Conclusion: A significant proportion of critically ill children receive at least one red blood cell transfusion during their PICU stay. Presence of anemia, cardiac disease, severe critical illness with multiple organ dysfunction syndromes are the most significant determinants of red blood cell transfusions in PICU.

Abbreviations: OR; odds ratio, $\mathrm{Cl}$; confidence interval.

843

\section{INTERFERON COMPARED WITH INF PLUS RIBAVIRIN FOR TREATMENT OF CHRONIC HEPATITIS C IN THALASSEMIA PATIENTS IN ALI ASGHAR CHILDREN'S HOSPITAL}

\author{
S. Ansari, F. Rezaie \\ Iran University of Medical Sciences, Tehran, Iran
}

Background: Hepatitis $C$ infection is common in transfusion dependent thalassemia .The effect of alpha interferon and INF plus ribavirin on hepatitis $\mathrm{C}$ was examined in patients.

The aim of the study was to assess the long term efficacy of two therapies and comparisons of efficacy of these .

Method: 23 patients had treated with alpha interferon and 14 patients had treated with INF plus ribavirin. INF was admitted three time a week (24-48 weeks)at dose $3 \mathrm{MIU} / \mathrm{m} 2$ and oral ribavirin $16 \mathrm{mg} / \mathrm{Kg} / \mathrm{d}$.We examined the serum HCV-RNA titer and ALT before and 6 month after treatment and comparison of both.

Results: The mean of serum level of ALT pretreatment of monotherapy was 102/95 and pretreatment of combination therapy was $84 / 75$.

The mean of serum level ALT 6 months after cessation of monotherapy was 95.45 and in combination therapy was 50.66 .

At 6 months after cessation of INF 5 patients had positive serum HCV -RNA, 7 patients had negative HCV-RNA and 11 patients had undetectable HCVRNA.

At 6 months after cessation of combination therapy, 5 patients had positive serum HCV-RNA, 1 patient had negative and 8 patients had undetectable HCVRNA.
Based on statistical information, the amount of response with INF treatment had no significant statistical difference compared with treatment combination therapy. Only the ratio of HCV-RNA before and after the treatment in patients with single therapy showed statistical difference. (Pvalue: 0.008)

Conclusion: INFmonotherapy proved to be an effective treatment, especially in thalassemia patients with chronic HCV in whom ribavirin cannot be used.

\section{4}

\section{EVALUATION OF IRON CHELATION THERAPY IN BETA THALASSEMIA MAJOR PATIENTS IN EAST DELTA OF EGYPT}

\author{
M. Badr, M. Hesham, T. Hassan, S.M. Badawy
}

Department of Pediatrics, Zagazig University, Zagazig, Egypt

Aims: Evaluation of the efficacy and safety of alternating deferiprone and desferrioxamine in reducing transfusional iron overload compared to either drug alone and to assess associations between compliance,complications and ferritin levels in thalassemic children in east delta of Egypt.

Methods: Retrospective-Prospective cohort study was carried out on 150 transfusion dependant thalassemic children on irrigular desferrioxamine chelation therapy.Patients were randomized into three groups(50 patients in each)according to iron chelation therapy regimen: Group I received deferiprone 5 days/week alternating with desferrioxamine for 2 days/week,group II received daily deferiprone only and group III received desferrioxamine 5 days/week.Efficacy, safety and tolerance of different chelation regimens were assessed periodicaly both clinicaly and laboratory. Results: There was highly significant reduction in serum ferritin levels after chelation therapy in all studied groups. The reduction was significantly higher in group I and group III than in group II especially in compliant patients. Patients of group II and group I were more compliant to chelation therapy than patients of group III. Regarding disease complications, hypogonadismandgrowthimpairment were the commonest disease complications in our study followed by osteoprosis and osteopenia. Complications were higher in non compliant patients and those with higher serum ferritin levels. Regarding 